\title{
DRENAGEM ENDOSCÓPICA DE ABSCESSO PANCREÁTICO
}

\section{ENDOSCOPIC DRAINAGE OF PANCREATIC ABSCESS}

\author{
Walton Albuquerque, TCBC-MG ${ }^{1}$ \\ Marcos Augusto de Carvalho ${ }^{2}$ \\ Edivaldo Fraga Moreira ${ }^{3}$
}

\section{INTRODUÇÃO}

O abscesso pancreático (AP) é uma das complicações da pancreatite aguda (PA) e a drenagem é imperativa para seu controle clínico. O padrão ouro para o tratamento desta complicação ainda é a drenagem cirúrgica tradicional, mas em face de sua elevada morbimortalidade a abordagem endoscópica criteriosa desta patologia vem ganhando espaço entre as várias formas de tratamento.

O objetivo do presente trabalho é mostrar nossa experiência inicial na abordagem endoscópica do AP mostrando que esta conduta é viável em casos selecionados.

\section{RELATO DOS CASOS}

\section{Caso 1}

RFB, 46 anos, sexo masculino, internado com quadro de PA associada à icterícia e febre. Ultra-sonografia abdominal (US) mostrou colecistolitíase. Submetido a colangiopancreatografia endoscópica retrógrada (CPER) seletiva para as vias biliares, esta não demonstrou coledocolitíase.

Submeteu-se a colecistectomia convencional e, alguns dias após, foi readmitido com dor em andar superior do abdome, febre e prostração. A tomografia computadorizada do abdome (TC) mostrou um pseudocisto de pâncreas comprimindo o arco duodenal e dilatação do colédoco.

A endoscopia digestiva alta (EDA) evidenciou compressão duodenal e área de exsudato na mucosa, que ao mínimo toque do aparelho drenou secreção purulenta. Por este local e já com o duodenoscópio de visão lateral e canal largo, foi introduzido um cateter e injetado o contraste iodado que documentou grande loja retroperitoneal.
Ampliado o trajeto transmural com dilatador hidrostático de $16 \mathrm{~mm}$ de diâmetro, houve drenagem ampla de pus. Foi então posicionado um dreno nasocístico e opacificado o Wirsung, que demonstrou desconexão completa na cabeça do pâncreas e que demandou a introdução de uma prótese transpapilar de $7 \mathrm{Fr}$.

A partir daí o paciente ficou assintomático. $\mathrm{O}$ controle por US três meses após evidenciou colabamento da loja, sendo trocada a prótese. Novamente com três meses, foi realizada colangiopancreatografia por ressonância magnética nuclear (CPRM) que não mostrou alterações, tendo sido retirada definitivamente a prótese, estando o paciente completamente assintomático.

\section{Caso 2}

HMS, 81 anos, sexo feminino, transferida para o nosso Hospital com quadro de coleção organizada peripancreática pós-pancreatite aguda biliar, diagnosticada pela TC, com dados clínicos e laboratoriais de infecção. Estava em uso de antibióticos de largo espectro, e apresentava sintomas de obstrução da saída gástrica. Submeteu-se a EDA que evidenciou volumoso abaulamento na parede posterior do antro gástrico, que foi puncionado com cateter de esclerose, e aspirado líquido purulento. Perfurada esta área com estilete ligado a eletrocautério e dilatado o trajeto com balão hidrostático, houve drenagem ampla de pus. Em seguida foi posicionado um dreno nasocístico na cavidade do abscesso.

A paciente recebeu alta hospitalar assintomática e no controle ambulatorial foi solicitado uma CPRM que mostrou pâncreas normal, colecistolitíase e ausência de coleções. Realizada colecistectomia videolaparoscópica sem intercorrências.

1. Chefe do Serviço de Endoscopia Digestiva do Hospital da Polícia Militar de Minas Gerais; Coordenador do Serviço de Endoscopia Digestiva do Instituto Alfa de Gastroenterologia do Hospital das Clínicas da Universidade Federal de Minas Gerais; Endoscopista dos Hospitais Felício Rocho e Governador Israel Pinheiro (IPSEMG), Belo Horizonte, MG.

2. Cirurgião Geral do Hospital Nossa Senhora de Lourdes, Nova Lima, MG. Assistente voluntário do Serviço de Endoscopia Digestiva do Hospital da Polícia Militar de Minas Gerais, Belo Horizonte, MG.

3. Coordenador do Serviço de Endoscopia Digestiva do Hospital Felício Rocho, Belo Horizonte, MG.

Recebido em 29/04/2002

Aceito para publicação em 13/08/2002

Trabalho realizado nos Hospitais Felício Rocho, Militar e Governador Israel Pinheiro (IPSEMG), Belo Horizonte, MG. 


\section{Caso 3}

EL, 57 anos, feminina, internada com quadro de coledocolitíase. Submetida a CPER e papilotomia endoscópica com retirada do cálculo que complicou com PA e pseudocisto volumoso em íntimo contato com a parede gástrica posterior, confirmado pela TC.

Realizada drenagem endoscópica da coleção retrogástrica com saída nítida de pus. Evoluiu com sangramento no local da punção gástrica, necessitando de cirurgia para controle do mesmo e drenagem externa da coleção pancreática. Deixada em laparostomia com revisões programadas, houve resolução completa do quadro abdominal. Evoluiu, no entanto, com piora do quadro clínico, vindo a falecer por insuficiência de múltiplos órgãos.

\section{DISCUSSÃO}

Tão importante quanto a drenagem dos abscessos pancreáticos é certificar-se da real etiopatogenia do processo: coleção peripancreática, necrose infectada, pseudocisto ou abscesso. Para o abscesso, o tratamento cirúrgico clássico foi, durante muitos anos, a única opção terapêutica. A drenagem endoscópica surgiu como opção em 1975, porém com altos índices de complicações (perfuração e sangramento). Dois avanços tecnológicos, aliados à maior experiência por parte dos endoscopistas, surgiram para melhorar esta abordagem: a ultra-sonografia endoscópica (USE) ${ }^{5}$ que permite localizar com precisão a coleção e puncioná-la diretamente, evitando perfurações inadvertidas, desviando de vasos maiores da parede gastrointestinal e afastando possíveis diagnósticos diferenciais, e o aprimoramento dos acessórios endoscópicos, como os dilatadores hidrostáticos passados pelo canal de trabalho dos aparelhos, que ampliam o orifício de drenagem, divulsionando os vasos em vez de cortá-los, minimizando hemorragias e permitindo drenar coleções espessas e debris. Diante disso, o índice de sangramento diminuiu, tornando esta abordagem uma alternativa terapêutica aceitável.

Para a drenagem endoscópica de coleção infectada é importante avaliar o grau de tecido desvitalizado pela TC com contraste. Quanto mais tecido sólido não perfundido (necrose), maior a indicação de tratamento cirúrgico clássico. Também, a presença de outra doença abdominal cirúrgica e/ou cistos multiloculados (complexos), pela possibilidade de degeneração maligna, indica a cirurgia tradicional. Alguns autores, entretanto, têm relatado o tratamento endoscópico para casos de necrose pancreática organizada $^{1-3}$. Baron ${ }^{3}$ tratou por endoscopia 11 pacientes com necrose pancreática organizada, tendo sucesso em nove deles. Existem critérios bem estabelecidos ${ }^{4}$ para a drenagem endoscópica de pseudocistos de pâncreas não infectados que devem ser seguidos rigorosamente também para as coleções infectadas, para minimizar as complicações, conforme Tabela 1.

A drenagem endoscópica pode ser realizada por via transpapilar (DTP), via transmural (DTM) ou combinada. A DTM é utilizada quando a coleção causa um abaulamento na parede de uma víscera oca, mais comumente o

\section{Tabela 1}

Princípios fundamentais para a drenagem endoscópica de coleções pancreáticas

Precisão diagnóstica

Paciente sintomático

Grande dimensão (>6cm)

Tempo de aparecimento acima de quatro semanas

Contíguo à parede gástrica ou duodenal

Distância menor que $1 \mathrm{~cm}$ à TC* ou USE**

Coleção uniloculada

*Tomografia computadorizada

**Ultra-sonografia endoscópica

estômago (gastrocistostomia) ou o duodeno (duodenocistostomia). A distância entre a coleção e a parede de tal víscera é importante e deve ser menor que $1 \mathrm{~cm}$ à TC ou USE. Nessa técnica, punciona-se o abaulamento com um estilete atingindo a coleção. Sob controle radioscópico, passa-se um fio-guia por esse pertuito e sobre este um balão hidrostático para dilatar o orifício, divulsionando os vasos ao invés de cortá-los (Figura 1A). Para manter a drenagem muitos optam pela colocação de uma prótese tipo biliar ou de um dreno nasocístico. A prótese tem o inconveniente de não permitir injeção de contraste e poder migrar, tanto para a luz gastrointestinal quanto para a cavidade cística, necessitar novo procedimento para retirá-la, além de poder obstruir diminuindo sua eficácia. $\mathrm{O}$ dreno nasocístico permite injeção de contraste para acompanhamento (Figura 1B), irrigação contínua de solução salina no caso de coleções espessas e sua retirada não requer novo procedimento. Tem como desvantagem o desconforto para o paciente e o risco de ser arrancado num momento de confusão mental, muito comum em doentes graves.

Em caso de comunicação da coleção com o ducto pancreático, pode-se optar pela drenagem transpapilar (DTP) realizada por cateterismo da papila duodenal maior, com ou sem papilotomia, contrastação do ducto pancreático e da coleção e passagem de prótese pancreática. Esse método tem menor risco de sangramento, porém pode não drenar adequadamente as coleções espessas, devido ao calibre fino da prótese pancreática. Ainda não há provas que a DTM associada a DTP (drenagem combinada) aumente o sucesso do método ${ }^{2}$, mas pode estar indicada quando o conteúdo do abscesso for espesso, em especial quando ocorre ruptura ductal, estenose ou cálculo distal ao mesmo.

Os resultados da drenagem a longo prazo dependem da etiologia da pancreatite, da morfologia ductal, da complexidade do abscesso e da gravidade da pancreatite. Portanto, deve-se considerar a alternativa endoscópica para casos de coleções pós-pancreatite, em íntimo contato com a parede gástrica ou duodenal, que obedeçam aos critérios de drenagem, particularmente em pacientes de risco cirúrgico elevado. 


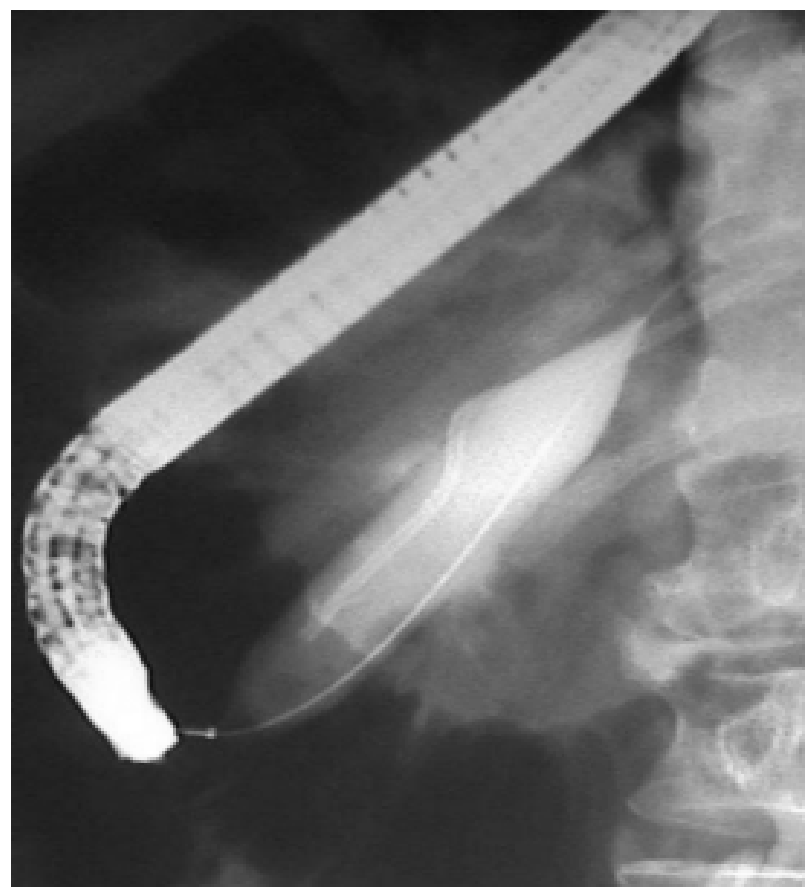

Figura 1A - Dilatação hidrostática do trajeto fistuloso entre o abscesso pancreático e o duodeno. Podemos observar uma endoprótese pancreática introduzida para melhorar a drenagem (drenagem combinada).

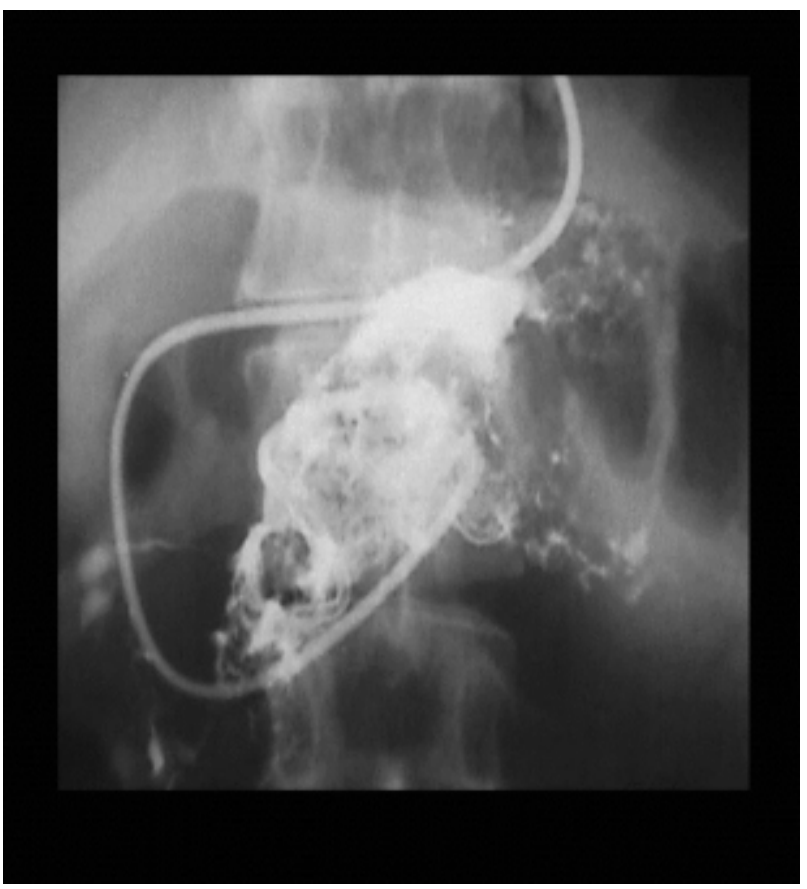

Figura 1B - Posicionamento do dreno nasocístico no abscesso pancreático retroduodenal, com opacificação da loja por contraste iodado.

\begin{abstract}
Surgical drainage is still considered the gold standard treatment of pancreatic abscess. Patients with high surgical risk, however, require alternative therapy. The authors report three cases of pancreatic abscess that were treated endoscopically. In patients who met endoscopic drainage criteria, treatment was effective, though one case did require surgical intervention as a result of gastric puncture point bleeding. After this initial experience, we believe that endoscopic drainage should be considered in selected cases.
\end{abstract}

Key Words: Pancreatic abscess; Endoscopic drainage

\title{
REFERÊNCIAS
}

1. Bruckner M, Grimm H, Nam VC et al. Endoscopic treatment of a pancreatic abscess originating from biliary pancreatitis. Surg Endosc, 1990, 4: 227-229.

2. Pinkas H, Dolan RP, Brady PG. Successful endoscopic transpapillary drainage of an infected pancreatic pseudocyst. Gastrointest Endosc, 1994, 40: 97-99.

3. Baron TH, Thaggard WG, Morgan DE et al. Endoscopic therapy for organized pancreatic necrosis. Gastroenterology, 1996, 111: 755-764.

4. Beckingham IJ, Krige JE, Bornman PC et al. Endoscopic management of pancreatic pseudocysts. Br J Surg, 1997, 84: 1638-1645.
5. Etzkorn KP, DeGuzman LJ, Holderman WH et al. Endoscopic drainage of pancreatic pseudocysts: patient selection and evaluation of the outcome by endoscopic ultrasonography. Endoscopy, 1995, 27: 329-333.

Endereço para correspondência:

Walton Albuquerque

Alameda Centauro, 298

34000-000 - Nova Lima-MG

E-mail:waltonendoscopia@uol.com.br 